\title{
SELECTIVE, PULSED CVD OF PLATINUM ON MICROFILAMENT GAS SENSORS
}

\author{
Ronald P. Manginell, James H. Smith, Antonio J. Ricco, Daniel J. Moreno, and Robert C. Hughes \\ Sandia National Laboratories
}

Albuquerque, NM 87185-1080

\author{
Robert J. Huber \\ University of Utah \\ Salt Lake City, UT 84112
}

\section{ABSTRACT}

A post-processing, selective micro-chemical vapor deposition ("micro-CVD") technology for the deposition of catalytic films on surface-micromachined, nitride-passivated polysilicon filaments has been investigated. Atmospheric pressure deposition of platinum on microfilaments was accomplished by thermal decomposition of platinum acetylacetonate, an organometallic Pt precursor; deposition occurs selectively only on those filaments which are electrically heated. Catalyst morphology, characterized by scanning electron microscopy, can be controlled by altering deposition time, filament temperature, and through the use of pulsed heating of the filament during deposition. Morphology plays an important role in determining the sensitivity of these devices when used as combustible gas sensors.

\section{INTRODUCTION}

Combustible gases such as methane and hydrogen pose a safety threat when they accumulate at explosive concentrations. A well-developed technology to detect such species in air is the use of an electrically heated Pt filament or Pt-loaded ceramic bead [13 ]. These catalytic gas sensors measure the temperature change induced in the catalyst during combustion by means of a resistance variation [4]. Despite successful implementation for many years, these conventional combustible gas sensors have several limitations. Due to their relatively large size, they have high power consumption and slow response time. Often, hand manufacture or hand sorting of these devices is required [5,6]. For these reasons, they can be expensive, unreliable and not reproducible. Our goal is to overcome these limitations by taking advantage of $\mathrm{Si}$ microfabrication technology. Furthermore, this method of fabrication allows for both improved selectivity through the development of sensor arrays, and the integration of electronics for control and data acquisition $[7,8]$.

Micromachined combustible gas sensors have been reported elsewhere [5-6,9-10]. Using surface micromachining and "micro-CVD" techniques, we have designed, modeled, fabricated, and tested Pt-coated polysilicon microfilaments for the calorimetric detection of combustible gas mixtures. Similar microfilaments have been previously explored as potential incandescent light sources and hot-wire anemometers [11,12] but have as yet to be exploited for chemical sensing. Our filament fabrication procedure is similar to that developed by Mastrangelo [13]. The filaments also have much in common with "micro-hotplates," [5,7,14-16] but offer the potential advantage of less thermal mass and consequent reductions in power consumption and response time.

This work focuses on a post-processing, selective "micro-CVD" technology for the deposition of catalytic films on silicon nitride-passivated polysilicon filaments (Figure 1). We

\author{
Stephen D. Senturia \\ Massachusetts Institute of Technology \\ Cambridge, MA 02139
}

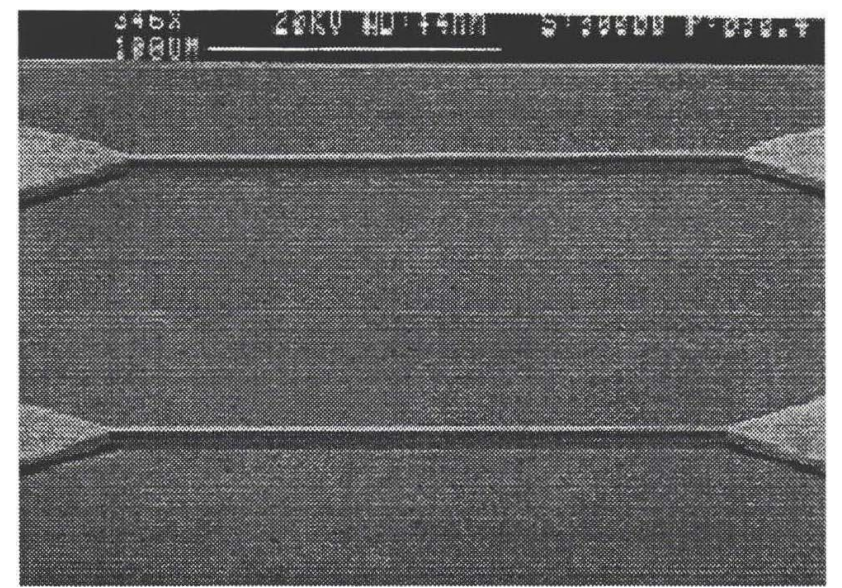

Figure 1. Near-profile SEM of 200 micron long filaments prior to $P t$ deposition The filaments are $2 \mu \mathrm{m}$ thick, $10 \mu \mathrm{m}$ wide and elevated $2 \mu \mathrm{m}$ above the substrate. They are encapsulated in a passivating layer of silicon nitride.

believe this is an important technology for many chemical microsensors, because (a) film deposition prior to release limits film choice due to possible attack by the etchant, and (b) the fragility of released microstuctures renders them unsuitable for many conventional deposition techniques [17]. We use atmospheric-pressure deposition of $\mathrm{Pt}$ on surface-micromachined microfilaments by thermal decomposition of an organometallic $\mathrm{Pt}$ precursor, platinum acetylacetonate. This post-packaging deposition occurs only on the selectively heated filament(s).

Because catalyst morphology plays a role in determining the sensitivity, selectivity, and longevity of combustible gas detectors, control of platinum film microstructure is critical. Scanning electron microscopy (SEM) studies of Pt films are presented here and show that control of film morphology can be achieved by altering deposition time, filament temperature, and importantly, through the use of pulsed heating of the filament during $\mathrm{Pt}$ deposition. Sensitivity of these devices to various concentrations of hydrogen in oxygen and nitrogen is also reported and shows that film morphology is a critical parameter.

The interplay between gas transport kinetics and surface reaction rates can affect the sensor response as a function of catalyst film morphology and, indeed, can influence the catalyst morphology during its deposition. Higher surface-area films contain a larger number of reaction sites, tending to drive the rate of surface reaction, and hence the heat deposited in the filament, towards the mass-transport limit, thus improving sensitivity to combustible mixtures. During the CVD process, the relatively larger accessible gas volume that supplies organometallic precursors to "sharp" filament features such as corners and edges yields a higher mass-transport-limited flux than in the planar 
regions of the filament, often resulting in edges covered by thicker and/or higher surface area deposits than found on top of the filament.

These variable-mass-transport effects can be manipulated by operation in a regime where depletion of the gas-phase concentrations of precursor (or reactants) is controlled: pulsed operation of the filament allows "replenishment time" between pulses, during which the concentrations of gaseous species recover to their bulk values. Manipulation of pulse width and duration therefore offers the opportunity to control the reaction rates for catalyst deposition, as well as combustible species detection, at various points between the two limits of mass transport and surface reaction rate.

\section{EXPERIMENT}

The micro-CVD apparatus is diagrammed in Figure 2. The packaged filament is placed in the CVD cell with a small amount of the Pt precursor. The cell is inserted in an oven at $140^{\circ} \mathrm{C}$. This temperature provides adequate vapor pressure of the $\mathrm{Pt}$ precursor in the cell, but no deposition occurs, except on those filaments that are electrically heated by the external control circuit. Deposition of platinum is optimized where filament temperature exceeds $450^{\circ} \mathrm{C}$ [18].

The control circuit is designed to maintain the filament at constant resistance and can provide continuous and pulsed reference resistances. In order to evaluate the effects of deposition time and filament bias mode (pulsed versus continuous), a matrix of deposition conditions was examined. For a given filament temperature distribution, deposition time and duty cycle of the heating pulse were varied. Deposition times of $0.7,1.5,3,7,15$ and $25 \mathrm{~min}$. were investigated using a $20 \mathrm{~Hz}$ pulse train with duty cycles of $5,10,20,50$ and $100 \%$ (continuous).

To detect combustible gas mixtures, the current and voltage required to maintain a Pt-coated filament at constant resistance were monitored during exposure to combustible hydrogen mixtures. Measurements were made in a steady-flow gas test bed in which total flow was regulated to $1 \mathrm{slm}$. Gas flows were prepared by mixing nitrogen and $20 \%$ oxygen with various concentrations of hydrogen using mass-flow controllers.

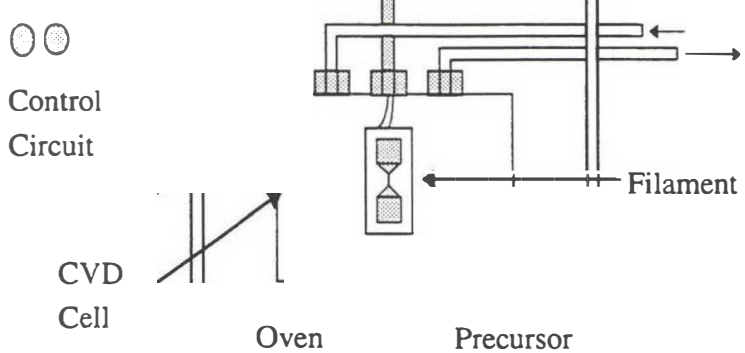

Figure 2. The micro-CVD apparatus: A teflon "CVD cell" houses the packaged filament and a small amount of the Pt precursor. The cell is equipped with electrical access to provide power to those filaments selected for deposition. Nitrogen lines insure an inert ambient in the cell. To vaporize the precursor, the CVD cell is inserted into an oven at $140^{\circ} \mathrm{C}$.

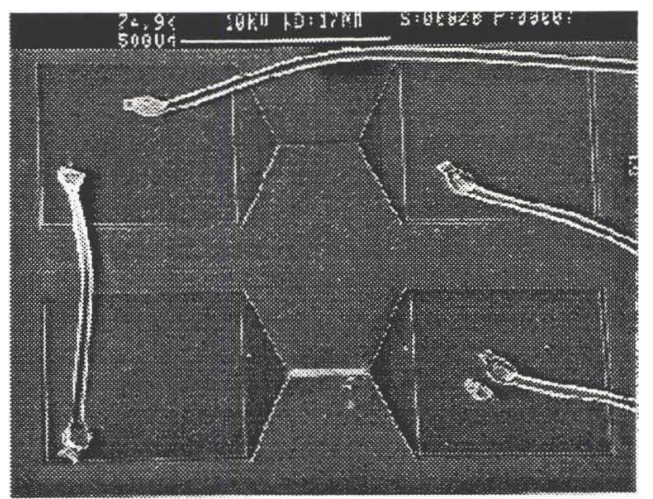

Figure 3. SEM top view of a pair of filaments. Al bond wires connect the square bond pads to a conventional dual in-line package (DIP). Only the lower filament which was electrically heated in the CVD cell contains a $0.2 \mu \mathrm{m}$ layer of $P t$

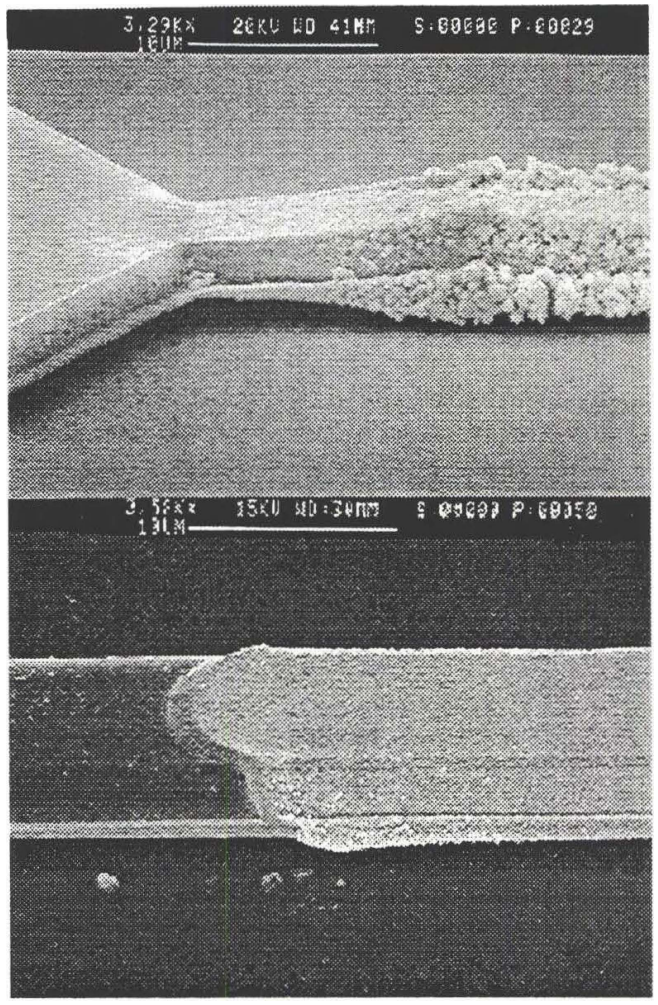

Figure 4. Near-profile SEMs of the ends of two filaments subjected to different deposition conditions. The high surface-area film on the top filament was deposited in continuous mode for 10minutes. The lower film underwent a 9\% duty-cycle $(5 \mathrm{msec}$ on, 50 msec off) deposition for a total elapsed time of $7 \mathrm{~min}$., and is relatively smooth in comparison to the upper film.

\section{RESULTS AND DISCUSSION}

Figure 3 shows a top view of side-by-side filaments, only one of which (the lower) was electrically heated and consequently coated with Pt. Figure 4 illustrates two extremes of catalyst morphology that can be obtained by varying the deposition time and the duty cycle of the heating pulse.

To better understand the role these factors play in controlling the morphology, one must make a fair comparison between films 
deposited under different conditions. One approach compares films formed using equivalent total energy inputs into the filaments during deposition. For a given filament temperature distribution, this energy is proportional to the amount of time the filament is heated during deposition (product of duty cycle and total deposition time). SEMs of two filaments, shown in Figure 5, demonstrate that although both had the same input energy, the higher duty-cycle heating increased the amount of Pt at that filament's edge. This result is consistent with the higher mass-ransport limit of catalyst precursor that is expected at the edges of these filaments. In the extreme case demonstrated in Figure 4, heavy deposition on the upper filament's edge threatens to thermally connect the device to the substrate, and thereby defeat its mode of operation. This scenario underlines the importance of film morphology control.

A slight increase in surface roughness with increased dutycycle is also evident in Figure 5, a result we have generally observed. Deposition time, on the other hand, mainly controls the film thickness, with a secondary effect on surface roughness. Figure 6 shows two devices with the same duty-cycle deposition (continuous), but different deposition times. An increase in film thickness with deposition time, along with an increase in roughness, is demonstrated.

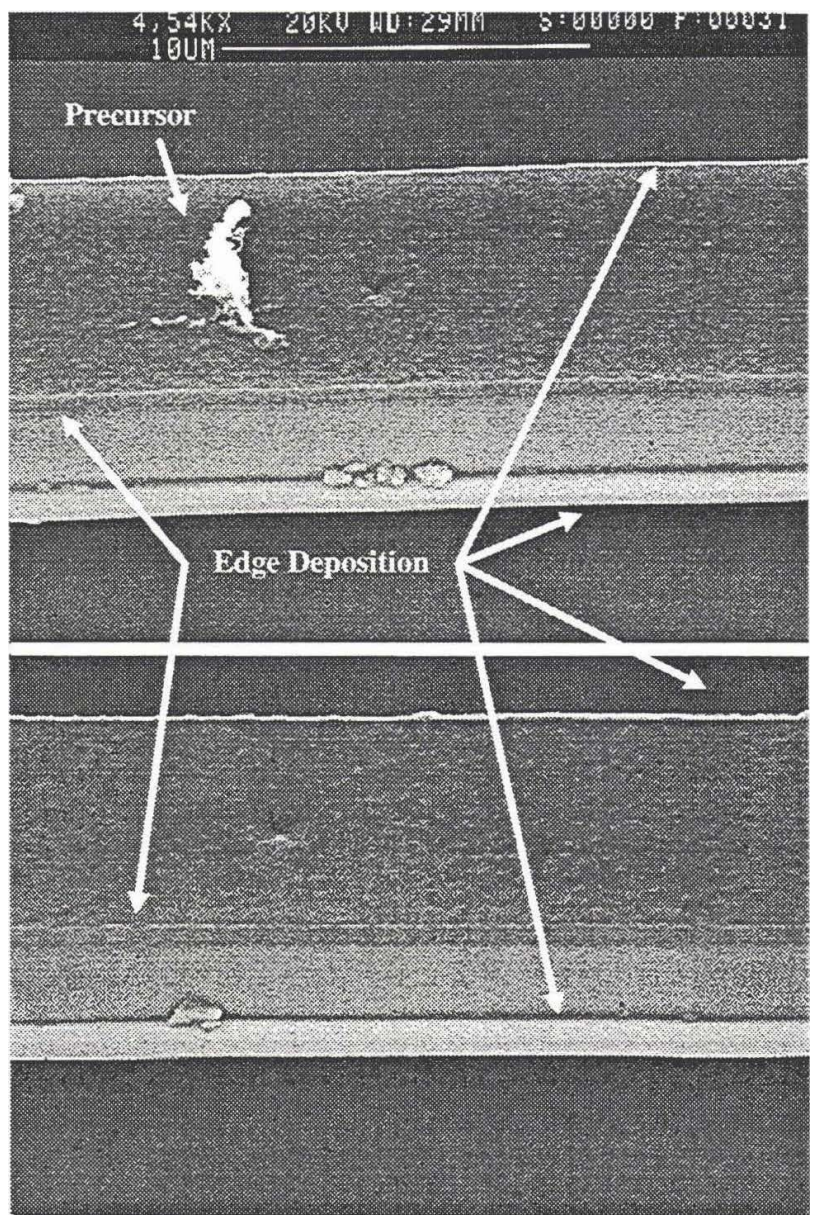

Figure 5. Two filaments with the same energy input. The upper filament underwent a $15 \mathrm{~min} .10 \%$ duty-cycle, $20 \mathrm{~Hz}$ deposition. The lower filament had a $7.5 \mathrm{~min}$. 20\% duty-cycle, $20 \mathrm{~Hz}$ deposition. The overall Pt surface roughness and Pt deposition on the filament edge have both increased with duty cycle. A particle of precursor remained on the upper filament subsequent to deposition. Both SEMs were taken at the same magnification

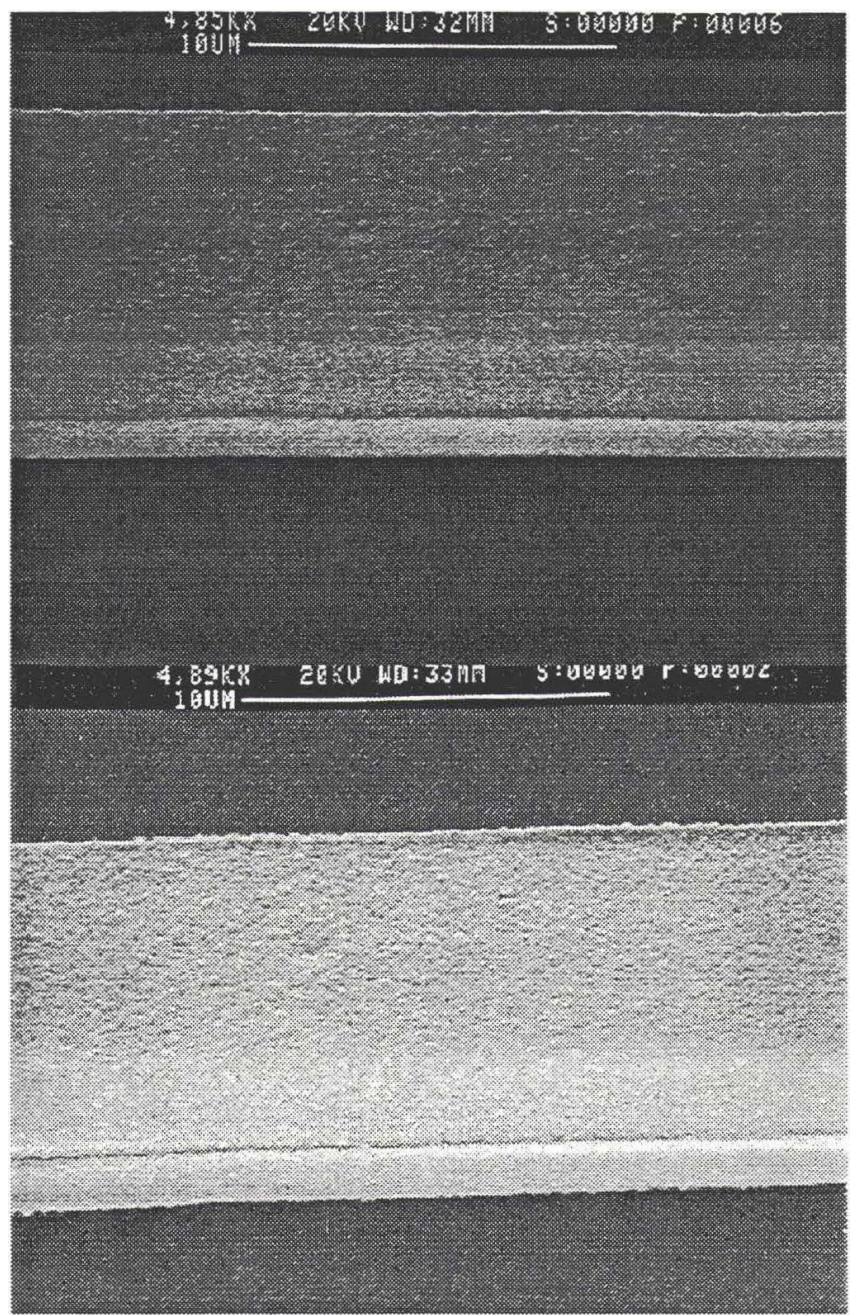

Figure 6. High magnification, plan view of two filaments. Both films were deposited in continuous mode. Deposition time and surface roughness increase from top to bottom in the figure. (From top to bottom, deposition times are 1.5 and $3.0 \mathrm{~min}$.)

Typical sensor response to mixtures of hydrogen in oxygen is given in Figure 7. These data demonstrate that for small gas concentrations, response is rapid (about 30 seconds for a full-scale response). Thermal response data show that the filament actually reaches operating temperature in $0.5 \mathrm{msec}$. The detection limit is $100 \mathrm{ppm}$ hydrogen in synthetic air. Furthermore, when operated in continuous mode, the filament consumes less than $70 \mathrm{~mW}$ of power; operation in a pulsed mode can further reduce total power consumption.

Figure 8 is a comparison plot, illustrating the relative effects of different deposition conditions (and therefore different catalyst morphologies) on sensor response. Prior to gas sensing, film morphology was evaluated qualitatively from SEM photographs. Film 1 had a smoother catalyst morphology and, as shown in Figure 8, roughly half the response of film 2. From these preliminary data it is clear that higher surface area films, which correspond to greater duty cycles and longer deposition times, are more sensitive. This result is consistent with mass-transport limited reaction rates. 


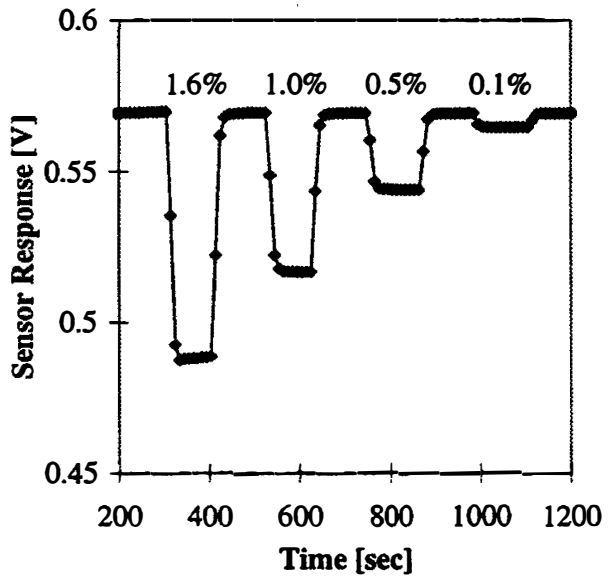

Figure 7. Sensor response of a Pt coated filament when subjected to various concentrations of hydrogen in $20 \%$ oxygen and a balance of nitrogen. The baseline signal is taken in pure nitrogen. Data was taken at $10 \mathrm{sec}$ per point.

\section{SUMMARY AND CONCLUSIONS}

We have demonstrated an atmospheric pressure, postprocessing, selective "micro-CVD" technology for the deposition of catalytic films on surface-micromachined, nitride-passivated polysilicon filaments. Catalyst morphology can be controlled by altering deposition time, filament temperature, and through the use of pulsed heating of the filament during deposition. Both deposition time and duty cycle can be varied to control the film thickness and surface roughness. Duty cycle also affects the amount of catalyst deposited at the filament edge. When used in the detection of combustion gases, these devices are low power, sensitive and fast. The most sensitive films have higher surface area, requiring longer duty cycles and deposition times. Future work will be directed at investigating the effects of filament temperature, precursor temperature, and pressure on morphology and sensitivity. By controlling these parameters, in addition to deposition time and duty cycle, film morphology and sensor response can be optimized.

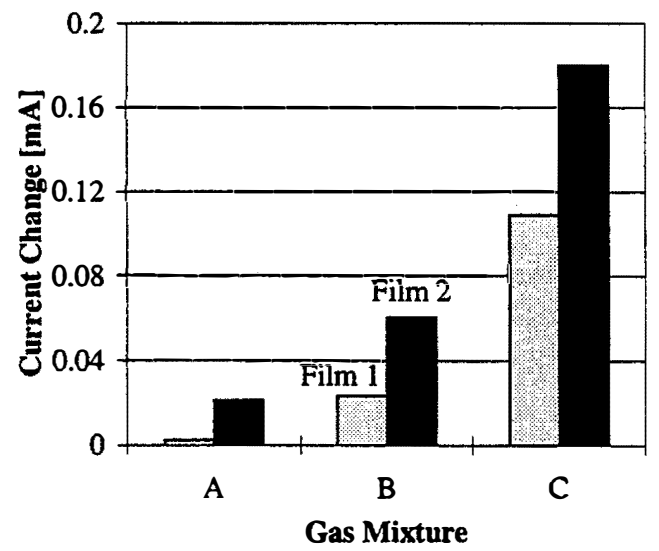

Figure 8. Response of two films, both deposited with a $20 \mathrm{~Hz}$ heating pulse, to different concentrations of hydrogen ( $A=0.5 \%$, $B=1 \%$ and $C=2 \%$ ) in $20 \%$ oxygen and a balance of nitrogen. Film 1 was deposited for 7 min. using a 10\% duty cycle pulse. Film 2 used a $20 \%$ duty-cycle, 7 min. deposition.

\section{ACKNOWLEDGMENTS}

The excellent technical assistance of A.W. Staton, M.W. Jenkins, M. Hill, and the engineers, operators and technicians of the Sandia Microelectronic Development Laboratory is gratefully acknowledged. Thanks also to P. Shea for the SEMs shown in this paper. This work, performed at Sandia National Laboratories, was supported by the U.S. Department of Energy under contract DEAC04-94AL85000.

\section{REFERENCES}

1. E. Jones, "The Pellistor Catalytic Gas Sensor", Solid State Gas Sensors, P.T. Moseleyand and BC. Tofield eds., A. Higler (Bristol and Philadelphia) 1987.

2. R. Dalla Betta and W.O. Byrnum, “A New Combustion Control Sensor”, Sensors, April 1989.

3. A. Accorsi, "Gas Sensors", Proc. Capteurs '86, Paris, France, 6/17-19/86, pp. 183-192.

4. S.J. Gentry and T.A. Jones, "The Role of Catalysis in SolidState Gas Sensors”, Sensors and Actuators, 10, 141 (1986).

5. M. Zanini, J. H. Visser, L. Rimai, R. Soltis, A. Kovalchuk, D. W. Hoffman, E. M. Logothetis, U. Bonne, L. Brewer, O. W. Bynum, and M. A. Richard, "Fabrication and Properties of a Sibased High Sensitivity Microcalorimetric Gas Sensor", Tech. Digest 1994 Sol.-State Sensor \& Actuator Workshop, Hilton Head Isl., SC, 6/13-16/94, pp. 176 - 79.

6. C. Vauchier, D. Charlot, G. Delapierre and A. Accorsi, "ThinFilm Gas Catalytic Microsensor", Sensors and Actuators B, 33 (1991).

7. R.E. Cavicchi, J.S. Suehle, P. Chaparala, K.G. Kreider, M. Gaitan, and S. Semancik, "Micro-hotplate Gas Sensor", Tech. Digest 1994 Sol.-State Sensor \& Actuator Workshop, Hilton Head Isl., SC, 6/13-16/94, pp. 53-56.

8. J. Smith, S. Montague, J. Sniewgoski, J. Murray, and P. McWhorter, "Embedded Micromechanical Devices for the Monolithic Integration of MEMS with CMOS", Proc. IEDM '95, pp. 609-612 (1995).

9. M. Gall, "The Si-Planar-Pellistor: A Low-power Pellistor Sensor in Si Thin-film Technlogy", Sensors and Actuators, B4, 553 (1991).

10. P. Krebs and A Grisel, "A Low Power Integrated Catalytic Gas Sensor”, Sensors and Actuators, B12, 155 (1993).

11. Y. C. Tai, R. S. Muller, and R. T. Howe, Transducers '85. 1985 International Conference on Solid-State Sensors and Actuators. Technical Digest of Papers, p. 445 (1985).

12. C. H. Mastrangelo and R. S. Muller, "A Constant Temperature Gas Flowmeter With A Silicon Micromachined Package", Tech. Digest 1988 Sol.-State Sensor and Actuator Workshop, Hilton Head Isl., SC, June, 1988, pp. 43 - 47. 
13. C.H. Mastrangelo, "Thermal applications of microbridges," Ph.D. Thesis, UC Berkeley, 1991.

14. C. L. Johnson, J. W. Schwank, and K. D. Wise, "Integrated Ultra-thin-film Gas Sensors", Sensors and Actuators B20, 55 (1994).

15. J. S. Suehle, R. E. Cavicchi, M. Gaitan, and S. Semancik, "Tin oxide gas sensor fabricated using CMOS micro-hotplates and in-situ processing", IEEE Electron Device Letters., 14, 118 (1993).

16. N. R. Swart and A. Nathan, "Design optimisation of integrated microhotplates", Sensors \& Actuators A, 43, 3 (1994).

17. S. Majoo, J.W. Schwank, J.L. Gland, and K.D. Wise, "A Selected-area CVD Method for Deposition of Sensing Films on Monolithically Integrated Gas Detectors", IEEE Electron Device Letters, 16, 217 (1995).

18. B.S. Kwak, P.N. First, A. Erbil, B.J. Wilkens, J.D. Budai, M.F. Chisholm, and L.A. Boamer, "Study of epitaxial platinum thin films grown by metalorganic chemical vapor deposition", $J$. Appl. Phys., 72, 3735 (1992). 